\title{
Corpora amylacea in adenocarcinoma of the prostate: incidence and histology within needle core biopsies
}

\author{
James D Christian, Tita C Lamm, John F Morrow and David G Bostwick \\ Bostwick Laboratories, Richmond, VA, USA
}

\begin{abstract}
Corpora amylacea in the prostate are a frequent finding in benign acini, but are only rarely observed in adenocarcinoma. To determine the incidence and comparative histopathology of this finding, we prospectively reviewed all consecutive needle core biopsies (excluding consultations) received at Bostwick Laboratories between December 2001 and July 2003. Among 5130 cases of adenocarcinoma (34\% of 15279 total needle biopsy cases), we identified 19 (31 biopsy specimens) with corpora amylacea within cancerous acini $(0.4 \%$ incidence). Patients ranged in age from 51 to 89 years (mean, 68 years). The corpora amylacea were located within cancers with Gleason pattern 3 (28 of 31 specimens), Gleason pattern 4 (one specimen), and Gleason pattern 5 (two specimens), and ranged from less than $0.1-0.3 \mathrm{~mm}$ in diameter. Coexistent eosinophilic proteinaceous debris was noted in all 31 specimens, luminal mucin in 19, crystalloids in 15, and collagenous micronodules in two specimens. Our results indicate that the incidence of corpora amylacea in adenocarcinoma is low, but the presence of such inclusions cannot be used to exclude malignancy.
\end{abstract}

Modern Pathology (2005) 18, 36-39, advance online publication, 13 August 2004; doi:10.1038/modpathol.3800250

Keywords: corpora amylacea; prostate; adenocarcinoma; needle core biopsies

Corpora amylacea are luminal secretions commonly present in benign prostatic acini, ${ }^{1-5}$ and have also been noted in other organs, including the lung and uterus. Corpora amylacea are present in the benign acini of prostates of approximately $25 \%$ of men aged 20-40 years; however, they are rare in carcinoma. ${ }^{6,7}$ In one study of prostatectomy specimens, corpora amylacea were found in up to $78 \%$ of benign acini and $11 \%$ of cancerous acini. ${ }^{5}$ Corpora amylacea may vary in size and shape, but are most frequently round. Their color ranges from pink-purple to orange, and the presence of concentric laminations is variable.

Corpora amylacea are thought to be related to epithelial cell desquamation and degeneration. ${ }^{6}$ Ultrastructurally, they are composed of bundles of fibrils and occasional interspersed electron-dense areas. ${ }^{8}$ Biochemical analysis and X-ray diffraction revealed that the main constituent of corpora amylacea was sulfated glycosaminoglycans. ${ }^{9}$ They are one of many intraluminal contents that can be found in prostatic acini, including mucin, crystal-

Correspondence: Dr JD Christian, MD, Bostwick Laboratories, 2807 North Parham Road, Richmond, VA 23294, USA.

E-mail: jchristian@bostwicklaboratories.com

Received 11 November 2003; revised 15 April 2004; accepted 8 July 2004; published online 13 August 2004 loids, and proteinaceous debris. Their high prevalence in benign acini and rarity in malignant ones may imply a benign diagnosis; this can be a diagnostic pitfall. To our knowledge, the incidence and comparative histology of corpora amylacea in adenocarcinoma has not been previously reported in needle core biopsy specimens.

\section{Materials and methods}

In all, 31 formalin-fixed microwave-processed needle biopsies (15 279 total needle biopsy cases) from 19 patients (5130 total cases of adenocarcinoma) were included for study. The biopsies were obtained prospectively between December 2001 and July 2003 from patients without prior treatment. The tissues were postfixed in Prefer solution (Anatech LTD, Battle Creek, MI, USA) and paraffin embedded. The original diagnosis in each case was made on hematoxylin and eosin-stained sections; eight cases were assisted by anti-keratin $34 \beta$-E12 immunohistochemical stains as per routine in our laboratory. All cases were prospectively identified and simultaneously reviewed, and a consensus formed by two or more pathologists.

The diagnosis, location, and presence of highgrade prostatic intraepithelial neoplasia were noted 
for each biopsy site, as well as the Gleason grade in the area of corpora amylacea. The number of cancer acini with corpora amylacea was recorded along with the presence or absence of laminations. The size of each cancer focus and diameter of the corpora amylacea and their respective cancerous acini were measured. Noncancer acini with corpora amylacea were evaluated at the same biopsy site (within one high-power field) and within the remainder of the benign tissue for each patient. The presence of other luminal and periluminal contents within individual acini was noted, including mucin, crystalloids, and collagenous micronodules. Proteinaceous debris within the acini was graded on a four-grade scale: minimal (less than $5 \%$ of acini contained debris), slight $(5-20 \%)$, moderate $(20-50 \%)$, and abundant (greater than 50\%). Accompanying acute and chronic inflammation within the adjacent stroma was scored on a three-grade scale: mild, moderate, and severe, as per routine in our laboratory.

\section{Results}

Among 15279 patients with 5130 cancers in prostate needle biopsies reviewed at Bostwick Laboratories from December 2001 to July 2003, a total of 19 cancer cases contained corpora amylacea $(0.4 \%$ incidence of cancers). Patients diagnosed with adenocarcinoma ranged in age from 51 to 89 years (mean, 68 years; median, 68 years). Serum PSA concentration was available in 16 patients (excluding cases 4, 13, and 19) and ranged from 3.8 to $11.7 \mathrm{ng} / \mathrm{ml}$ (mean, $7.0 \mathrm{ng} / \mathrm{ml}$; median, $6.1 \mathrm{ng} / \mathrm{ml}$ ).

\section{Pathologic Findings}

Of the 31 needle biopsy specimens with corpora amylacea, adenocarcinoma was Gleason score $3+$ $3=6$ in $25,3+4=7$ in two, $4+3=7$ in one, and $4+$ $5=9$ in three. Of these patterns, corpora amylacea were predominantly located within Gleason pattern 3 (28 of 31 specimens). In the remaining three specimens, the corpora amylacea were found in Gleason pattern 4 (one) and Gleason pattern 5 (two). Cancer foci ranged from 0.4 to $4.0 \mathrm{~mm}$ (mean, $1.9 \mathrm{~mm}$; median, $2.0 \mathrm{~mm}$ ) in greatest dimension and were located on the right side in $73 \%$ of specimens designated by location (one case was not separated by location). Of the 285 cancerous acini with corpora amylacea, 108 contained corpora amylacea with laminations. Corpora amylacea in cancer acini ranged from less than $0.1-0.3 \mathrm{~mm}$ in diameter; corresponding acinar size ranged from less than $0.1-0.4 \mathrm{~mm}$ in diameter. High-grade prostatic intraepithelial neoplasia was seen in $19 \%$ of the specimens with a concomitant diagnosis of adenocarcinoma; however, corpora amylacea were not found in any of the glands with high-grade prostatic intraepithelial neoplasia. Eosinophilic proteinaceous debris was present in minimal to abundant amounts in all 31 cases; luminal mucin was mixed with corpora amylacea in 19 of 31 specimens, crystalloids in 15 of 31 specimens (seven contained crystalloids and corpora amylacea in the same acinus), and collagenous micronodules in two of 31 specimens. None of the corpora amylacea found in the cases of adenocarcinoma showed calcification. Of the 31 biopsy specimens with adenocarcinoma, 22 also contained corpora amylacea within benign acini at the same site. Accompanying mild acute and chronic inflammation were present within one high-power field in 6 and $16 \%$ of the specimens with corpora amylacea, respectively.

\section{Discussion}

We found that corpora amylacea were present in a small but significant number of cases of adenocarcinoma. The corpora amylacea were located in malignant acini within predominantly Gleason pattern 3 (28 of 31 cases; the remaining three located in Gleason pattern 4 (one) and Gleason pattern 5 (two)) (Figure 1). The overall incidence of corpora amylacea in cancerous acini in this needle biopsy study was $0.4 \%$, compared to $11 \%$ in a separate prostatectomy study ${ }^{5}$ and $13 \%$ of cancerous acini in a study involving transurethral resection specimens. ${ }^{10}$ Corpora amylacea have been reported in $55 \%$ of cases of high-grade prostatic intraepithelial neoplasia in radical prostatectomy specimens. ${ }^{11}$ Our results may differ owing to sampling variation between the needle core biopsy specimens in the current study as compared to the radical prostatectomy and transurethral resection specimens.

Corpora amylacea were reported in $32 \%$ of cases of atypical adenomatous hyperplasia (AAH), ${ }^{10} 75 \%$ of cases of postatrophic hyperplasia, ${ }^{12}$ as well as in $20 \%$ of needle biopsies with infarcts, supporting the benign character of infarction. ${ }^{13}$ In contrast, corpora amylacea were absent in prostatic cancer in postradiation needle biopsies. ${ }^{14,15}$ This finding has been advanced as an aid for benignancy in postirradiation cases in which the cytologic features are unreliable. Also, corpora amylacea can be used to distinguish between adenocarcinoma and cases of atypical small acinar proliferation suspicious for but not diagnostic of malignancy (ASAP); we previously noted that corpora amylacea were not present in 48 cases of ASAP diagnosed among 1009 prostate needle biopsy specimens. ${ }^{16}$ Other luminal contents support a diagnosis of malignancy.

Acidic sulfated and nonsulfated mucin is present in acini of prostatic adenocarcinoma in up to $52 \%$ of cases ${ }^{17}$ appearing as amorphous or delicate threadlike faintly basophilic secretions in routine sections. Such mucins stain with alcian blue, and are best demonstrated at $\mathrm{pH} 2.5$, whereas the benign prostatic epithelium contains periodic acid Schiffreactive neutral mucin. Acidic mucin is not specific for carcinoma, and may be found in prostatic 

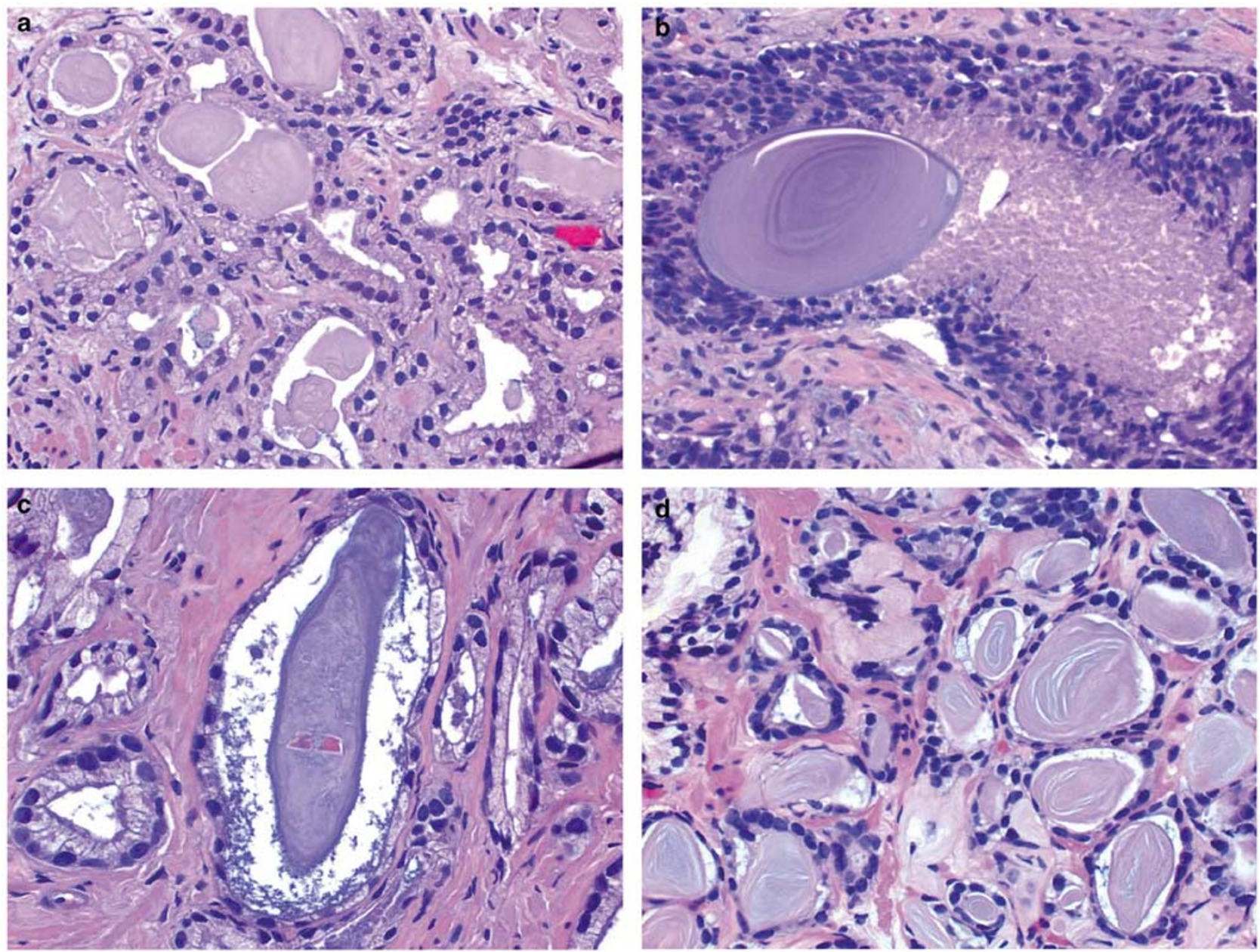

Figure 1 Histologic spectrum of corpora amylacea in adenocarcinoma. (a) Corpora amylacea within acini of Gleason pattern 3 adenocarcinoma. (b) Corpus amylaceum with accompanying necrosis within Gleason pattern 5 adenocarcinoma. (c) Crystalloids and corpus amylaceum in the same acinus. (d) Corpora amylacea with coexistent collagenous micronodules.

intraepithelial neoplasia (PIN), atypical adenomatous hyperplasia (AAH), sclerosing adenosis, and, rarely, in nodular hyperplasia. ${ }^{18-22}$ In the current study, luminal mucin was noted in $62 \%$ of cancer biopsies with corpora amylacea.

Crystalloids are sharp needle-like eosinophilic structures which are often present in the lumens of well-differentiated (36\%) and moderately differentiated cancer $(14 \%){ }^{23,24}$ They are not specific for carcinoma, and can be found in atypical adenomatous hyperplasia, PIN, nodular hyperplasia, and benign prostatic epithelium. In the current study, crystalloids were present in $42 \%$ of cancers in needle biopsy specimens which also contained corpora amylacea $(47 \%$ of these contained crystalloids and corpora amylacea in the same acinus).

Collagenous micronodules are a specific but infrequent and incidental finding in prostatic adenocarcinoma, consisting of microscopic nodular masses of paucicellular eosinophilic fibrillar stroma which impinge on acinar lumens. ${ }^{25,26}$ They are usually present in mucin-producing adenocarcinoma, and result from extravasation of acidic mucin into the stroma. Collagenous micronodules are present in about $13 \%$ of cases of adenocarcinoma, and are not observed in benign epithelium, nodular hyperplasia, or PIN. They are an infrequent finding, present in $0.6 \%$ of needle biopsies and $12.7 \%$ of prostatectomies. ${ }^{26}$ Collagenous micronodules are a useful but infrequent diagnostic clue in prostatic adenocarcinoma, and may be particularly valuable in challenging needle biopsy specimens. ${ }^{26}$ In the current study, we found collagenous micronodules in $6 \%$ of cancers containing corpora amylacea.

Our study is limited by reliance on the lightmicroscopic features of corpora amylacea within adenocarcinoma for identification of cases, as well as partial sampling of specimens by biopsy, and thus probably underestimates the true incidence of corpora amylacea in adenocarcinoma. Also, we employed microwave-processed specimens, and this form of tissue processing may result in a greater or lower incidence of corpora amylacea than other more conventional methods.

In summary, we found that corpora amylacea are histologically rare in cases of adenocarcinoma, but 
their presence should not be used as a criterion for exclusion of a malignant entity.

\section{References}

1 Eastman JR. The origin of corpora amylacea in the prostate gland. JAMA 1897;29:158-162.

2 Moore RA, Hanzel BF. Chemical composition of prostatic corpora amylacea and calculi. Arch Pathol 1936;22:41-54.

3 Moore RA. Morphology of prostatic corpora amylacea and calculi. Arch Pathol 1936;22:24-40.

4 Seaman AR. Cytochemical observations on corpora amylacea of human prostate gland. J Urol 1956;76:99-106.

5 Humphrey PA, Vollmer RT. Corpora amylacea in adenocarcinoma of the prostate: prevalence in 100 prostatectomies and clinicopathologic correlations. Surg Path 1990;3:133-141.

6 Marx AJ, Moskal JF, Gueft B. Prostatic corpora amylacea. A study with the electron microscope and electron probe. Arch Pathol 1965;80:487-494.

7 Andrews GS. The histology of the human foetal and prepubertal prostates. J Anat 1951;85:44-54.

8 Drachenberg CB, Papadimitriou JC. Prostatic corpora amylacea and crystalloids: similarities and differences on ultrastructural and histochemical studies. J Submicrosc Cytol Pathol 1996;28:141-150.

9 Cohen RJ, McNeal JE, Redmond SL, et al. Luminal contents of benign and malignant prostatic glands: correspondence to altered secretory mechanisms. Hum Pathol 2000;31:94-100.

10 Bostwick DG, Srigley J, Grignon D, et al. Atypical adenomatous hyperplasia: morphologic criteria for its distinction from well-differentiated carcinoma. Hum Pathol 1993;24:819-832.

11 Bostwick DG, Amin MB, Dundore P, et al. Architectural patterns of high-grade prostatic intraepithelial neoplasia. Hum Pathol 1993;24:298-310.

12 Cheville JC, Bostwick DG. Postatrophic hyperplasia of the prostate. A histologic mimic of prostatic adenocarcinoma. Am J Surg Pathol 1995;19:1068-1076.

13 Milord RA, Kahane H, Epstein JI. Infarct of the prostate gland: experience on needle biopsy specimens. Am J Surg Pathol 2000;24:1378-1384.
14 Cheng L, Cheville JC, Bostwick DG. Diagnosis of prostate cancer in needle biopsies after radiation therapy. Am J Surg Pathol 1999;23:1173-1183.

15 Bostwick DG, Qian J, Schlesinger C. Contemporary pathology of prostate cancer. Urol Clin North Am 2003;30:181-207.

16 Cheville JC, Reznicek MJ, Bostwick DG. The focus of 'atypical glands, suspicious for malignancy' in prostatic needle biopsy specimens: incidence, histologic features, and clinical follow-up of cases diagnosed in a community practice. Am J Clin Pathol 1997;108: 633-640.

17 Varma M, Lee MW, Tamboli P, et al. Morphologic criteria for the diagnosis of prostatic adenocarcinoma in needle biopsy specimens. A study of 250 consecutive cases in a routine surgical pathology practice. Arch Pathol Lab Med 2002;126:554-561.

18 Ro JY, el-Naggar A, Ayala AG, et al. Signet-ring-cell carcinoma of the prostate. Electron-microscopic and immunohistochemical studies of eight cases. Am J Surg Pathol 1988;12:453-460.

19 Ro JY, Grignon DJ, Troncoso P, et al. Mucin in prostatic adenocarcinoma. Semin Diagn Pathol 1988;5:273-283.

20 Humphrey PA. Mucin in severe dysplasia in the prostate. Surg Pathol 1991;4:137-143.

21 Epstein JI, Fynheer J. Acidic mucin in the prostate: can it differentiate adenosis from adenocarcinoma? Hum Pathol 1992;23:1321-1325.

22 Goldstein NS, Qian J, Bostwick DG. Mucin expression in atypical adenomatous hyperplasia of the prostate. Hum Pathol 1995;26:887-891.

23 Holmes EJ. Crystalloids of prostatic carcinoma: relationship to Bence-Jones crystals. Cancer 1977;39: 2073-2080.

24 Del Rosario AD, Bui HX, Abdulla M, et al. Sulfur-rich prostatic intraluminal crystalloids: a surgical pathologic and electron probe x-ray microanalytic study. Hum Pathol 1993;24:1159-1167.

25 McNeal JE, Alroy J, Villers A, et al. Mucinous differentiation in prostatic adenocarcinoma. Hum Pathol 1991;22:979-988.

26 Bostwick DG, Wollan P, Adlakha K. Collagenous micronodules in prostate cancer. A specific but infrequent diagnostic finding. Arch Pathol Lab Med 1995;119:444-447. 